\title{
Ultrasound or near-infrared vascular imaging to guide peripheral intravenous catheterization in children: a pragmatic randomized controlled trial
}

\author{
Sarah J. Curtis MD MSc, William R. Craig MDCM MSc, Erin Logue MN, Ben Vandermeer MSc, \\ Amanda Hanson MD, Terry Klassen MD MSc
}

\begin{abstract}
Background: Peripheral intravenous catheterization in children is challenging, and success rates vary greatly. We conducted a pragmatic randomized controlled trial to determine whether the use of ultrasound or near-infrared vascular imaging to guide catheterization would be more effective than the standard approach in achieving successful catheter placement on the first attempt.

Methods: We enrolled a convenience sample of 418 children in a pediatric emergency department who required peripheral intravenous catheterization between June 2010 to August 2012. We stratified them by age ( $\leq 3 \mathrm{yr}$ and $>3 \mathrm{yr}$ ) and randomly assigned them to undergo the procedure with the standard approach, or with the help of either ultrasound or near-infrared vascular imaging. The primary outcome was the proportion of patients who had successful placement of a catheter on the first attempt.
\end{abstract}

Results: The rate of successful first attempts did not differ significantly between either of the 2 intervention groups and the standard approach group (differences in proportions -3.9\%, 95\% confidence interval $[\mathrm{Cl}]-14.2 \%$ to $6.5 \%$, for ultrasound imaging; $-8.7 \%, 95 \% \mathrm{Cl}-19.4 \%$ to $1.9 \%$, for near-infrared imaging). Among children 3 years and younger, the difference in success rates relative to standard care was also not significant for ultrasound imaging $(-9.6 \%, 95 \%$ $\mathrm{Cl}-29.8 \%$ to $10.6 \%$ ), but it was significantly worse for near-infrared imaging $(-20.1 \%, 95 \%$ $\mathrm{Cl}-40.1 \%$ to $-0.2 \%)$. Among children older than 3 years, the differences in success rates relative to standard care were smaller but not significant $(-2.3 \%, 95 \% \mathrm{Cl}-13.6 \%$ to $9.0 \%$, for ultrasound imaging; $-4.1 \%, 95 \% \mathrm{Cl}-15.7 \%$ to $7.5 \%$, for near-infrared imaging). None of the pairwise comparisons were statistically significant in any of the outcomes.

Interpretation: Neither technology improved first-attempt success rates of peripheral intravenous catheterization in children, even in the younger group. These findings do not support investment in these technologies for routine peripheral intravenous catheterization in children. Trial registration: ClinicalTrials.gov, no. NCT01133652.
$\mathrm{W}$ hen painful procedures such as peripheral intraveneous catheterization are necessary for the medical care of children, the patients and their families want the first attempt to be successful. ${ }^{1}$ Success rates of first attempt at intravenous catheter placement in children vary greatly, from $40 \%-76 \%$, with up to 15 attempts reported in one case. ${ }^{2-9}$ Failed procedures ensure further painful skin punctures, significant delays in urgent treatment, decreased efficiency and increased costs to the health care system. ${ }^{10,11}$

The standard procedure for peripheral intravenous catheterization involves placement of the needle in a location considered most likely to contain a vein, with the choice based on visualization and palpation of the vessel, knowledge of anatomy and experience of the practitioner, most often a nurse. Venous access in children can be challenging. Sick children may have low circulating blood volumes and may be cold or in shock, all of which can lead to peripheral vasoconstriction. Furthermore, young infants and toddlers have small vessels, often obscured by subcutaneous fat, and are less able to cooperate with painful procedures. The stress from struggling, crying and fear results in additional peripheral vasoconstriction of already small vessels due to sympathetic overdrive.

Two adjunct technologies to facilitate intravenous catheterization in children have looked promising. Ultrasonography offers visual information about the size and depth of blood vessels, potentially facilitating intravenous placement of
Competing interests: None declared.

This article has been peer reviewed.

Correspondence to:

Sarah Curtis,

scurtis@ualberta.ca

Accepted: Mar. 24, 2015

Published: Apr. 20, 2015

CMAJ 2015. DOI:10.1503 /cmaj.141012 
the needle in real time. Two small randomized trials of ultrasound imaging for intravenous placement in a pediatric emergency department setting have been published, but both had physicians as the ultrasonographers. ${ }^{12,13}$ Near-infrared imaging devices project near-infrared light onto the skin, which is absorbed by deoxygenated hemoglobin. The invisible image of the underlying vascular pattern is captured by the device, processed and projected, in real time, back onto the patient's skin using visible green light. This technology allows hands-free visualization of a vascular map to guide catheter placement.

We performed a pragmatic randomized controlled trial to determine whether the use of either ultrasound or near-infrared vascular imaging would significantly improve the success rate of peripheral intravenous catheterization in children on first attempt by nurses compared with the standard approach.

\section{Methods}

\section{Study design and setting}

We conducted a stratified, parallel-group, randomized controlled trial to compare the use of ultrasound or near-infrared vascular imaging with the standard approach to achieve peripheral intravenous catheterization on the first attempt in children seen in the emergency department at the Stollery Children's Hospital, Edmonton, between June 2010 and August 2012. The hospital is the tertiary referral centre for northern Alberta, with 45000 visits to the pediatric emergency department annually.

Ethics approval for the study was obtained from the Health Research Ethics Board at the University of Alberta. The ultrasound and nearinfrared devices used in the trial had previously been approved for clinical use in Canada.

\section{Participants}

We included participants from a convenience sample of all eligible children aged 16 or younger who required peripheral intravenous catheterization as part of routine care and whose primary nurse agreed to participate in the study. Research assistants were available 50 hours per week to screen for potentially eligible patients and obtain consent from them or their parents. Children over the age of 7 years were asked to give written assent or oral assent if the child was unable to sign (e.g., broken arm); written consent was obtained from the parents of all children. We excluded patients if they were in critical condition, if the child's or parents' knowledge of English was insufficient for consent, or if the procedural nurse declined trial participation.

\section{Interventions}

The VeinViewer GS (Luminetx Corp.) was used for the near-infrared vascular imaging. Ultrasonography was performed with the M-Turbo Ultrasound System (SonoSite Inc.), with a standard $25-\mathrm{mm}$ broadband linear array and $6-13 \mathrm{MHz}$ transducer. Before the start of the trial, all emergency department nurses were invited to participate in the trial. Those who accepted were instructed on how to use both devices for intravenous catheterization. Training took 3 hours and included a lecture explaining technological constructs, practical applications and the manufacturer's instructions, as well as one-on-one training with a research assistant until the nurse affirmed confidence with both technologies for intravenous catheterization. For ultrasound training, nurses also received a self-study instructional booklet containing detailed illustrations of ultrasound techniques for intravenous catheterization and hands-on sessions using ultrasound for catheterization in artificial limbs. In addition, self-directed, hands-on practice with both devices was available for 2 weeks before the trial started.

\section{Protocol}

Once eligibility, informed consent and assent were confirmed, participants were randomly assigned, in the appropriate age stratum, to 1 of 3 groups: peripheral intravenous catheterization with the standard approach or with the help of either ultrasound or near-infrared vascular imaging. Randomization was achieved with the use of an online, computer-generated program. An agestratified ( $\leq 3 \mathrm{yr}$ and $>3 \mathrm{yr}$ ) block-randomization scheme (alternating blocks of 6 and 9) was used. We chose the age of 3 years as the cut-off point, because thereafter subcutaneous fat is reduced, children are more amenable to distraction and preparation for the procedure, and blood vessels are larger. Thus, we expected the challenges of intravenous catheterization to be different across these 2 age strata.

All procedures were performed in private treatment rooms under standard fluorescent lighting. Standard techniques to facilitate intravenous catheterization were used. The research assistant recorded baseline and multiple prospective outcome variables. Times were recorded by stopwatch. Intravenous catheterization was attempted until it was either successful or was abandoned as per routine clinical decision-making.

All data were entered into the Web-based electronic data-capture system OpenClinica. A study log documented patients who were excluded, missed or had refused to participate.

Because of the physical nature of the interventions, it was not possible to conceal the group 
allocation from the research assistants, nurses or trial participants. The randomization code was maintained by the Clinical Research Informatics Core at the Women and Children's Research Institute, University of Alberta, and was not revealed to the research team and statistician until data entry and analyses were completed.

\section{Outcome measures}

The primary outcome measure was the proportion of patients who had successful intravenous catheterization on the first attempt. We chose this patient-centred outcome measure rather than "overall success" because successful placement of the catheter on first attempt matters most to the patients and their families. This measure also captures system aspects, because failure directly implies prolonged procedural times and increased costs. We defined a successful attempt as clear flush through the catheter with $5 \mathrm{~mL}$ of normal saline without extravasation.

Secondary outcomes included the number of attempts to successful placement and the time to successful placement in minutes.

\section{Statistical analysis}

Previous research reported first-attempt success rates of about $70 \%$ for standard intravenous catheterization in pediatric emergency departments. ${ }^{6-8}$ We decided that an increase in this rate to $85 \%$ with the use of either technology would be clinically significant. We determined that a sample of 418 patients (about 140 in each group) would allow us to detect this difference with $80 \%$ power, assuming a 2 -sided test and a significance level $(\alpha)$ of $5 \%$. This sample size also allowed us flexibility to examine the effectiveness of each method by age strata.

All data were analyzed by intention-to-treat analysis. Analysis was performed with the use of either SAS (version 9.3, SAS Institute Inc.) or StatXact (version 10.0, Cytel). We computed proportions for dichotomous data and compared them using the Fisher-Freedman-Halton test. We computed means \pm standard deviations and medians with ranges for continuous data and compared them using either the Kruskal-Wallis test or oneway analysis of variance. We considered a $p$ value of less than 0.05 to be statistically significant.

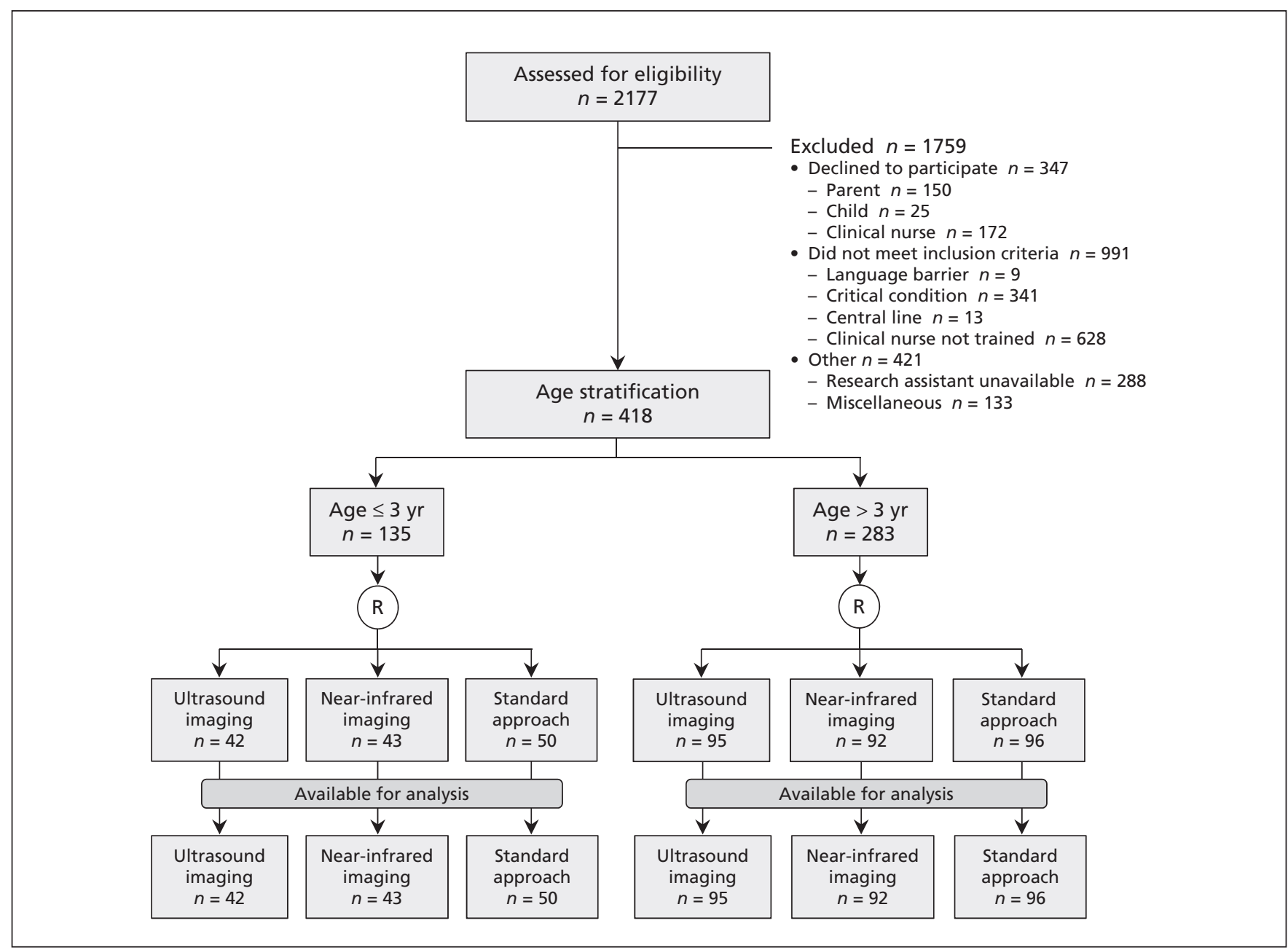

Figure 1: Selection of patients and allocation to study arms. No patients were excluded from analysis or lost to follow-up. $R=$ randomization. 
We computed differences between the intervention and standard approach groups using relative risks and differences in proportions (with 95\% confidence intervals [CIs]) for binary outcomes, and mean differences (with 95\% CIs) for continuous data. We performed a priori logistic regression analyses using binary logit models to test whether nursing characteristics, patients' body mass index (BMI) and group allocation were associated with successful catheterization on first attempt.

We conducted a sensitivity analysis on the primary outcome using the Wald test to take into account the clustering effect of some nurses having performed the procedure on several patients.

\section{Funding}

This research was funded by the Women and Children's Health Research Institute through support from the Stollery Children's Hospital Foundation. The manufacturers of the ultrasound and near-infrared technologies used in the study did not supply the devices for the trial. Neither the funding agency nor the manufacturers of the devices played a role in the design or conduct of the study, the interpretation of the results, the manuscript preparation or the decision to publish.

\section{Results}

We enrolled a total of 418 patients in the trial; 135 were 3 years or younger, and 283 were older than 3 years. Data from all patients were available for analysis in the groups to which they were initially assigned (Figure 1). Baseline characteristics were similar across the study arms (Table 1).

\section{Effect on first-attempt success rates}

The overall success rate of intravenous catheterization on first attempt did not differ significantly between either of the 2 intervention groups and the standard approach group (difference in proportion $-3.9 \%, 95 \% \mathrm{CI}-14.2 \%$ to $6.5 \%$, for ultrasound imaging; $-8.7 \%, 95 \%$ CI $-19.4 \%$ to $1.9 \%$, for near-infrared imaging) (Table 2). When stratified by age, the differences were generally not significant. Among children 3 years and younger, the difference in proportions between ultrasound-guided catheterization and the standard approach was not significant $(-9.6 \%, 95 \% \mathrm{CI}$ $-29.8 \%$ to $10.6 \%$ ), but it was significantly worse for near-infrared imaging relative to standard care $(-20.1 \%, 95 \%$ CI $-40.1 \%$ to $-0.2 \%)$. Among children older than 3 years, the differences in proportions were not significant relative to standard care $(-2.3 \%, 95 \% \mathrm{CI}-13.6 \%$ to $9.0 \%$, for ultrasound imaging; $-4.1 \%, 95 \% \mathrm{CI}-15.7 \%$ to $7.5 \%$, for near-infrared imaging). None of the pairwise

\begin{tabular}{|c|c|c|c|}
\hline Characteristic & $\begin{array}{l}\text { Ultrasound } \\
\text { imaging } \\
n=137\end{array}$ & $\begin{array}{l}\text { Near-infrared } \\
\text { vascular imaging } \\
\quad n=135\end{array}$ & $\begin{array}{c}\text { Standard } \\
\text { approach } \\
n=146\end{array}$ \\
\hline \multicolumn{4}{|l|}{ Age, yr } \\
\hline Mean \pm SD & $7.81 \pm 5.71$ & $7.20 \pm 5.75$ & $6.76 \pm 5.41$ \\
\hline Median (range) & 7.00 (0.07 to 16.90$)$ & 6.27 (0.02 to 16.98$)$ & 5.95 (0.00 to 16.74$)$ \\
\hline Male sex, \% $(n / N)$ & $54.0(74 / 137)$ & $47.4(64 / 135)$ & $57.9(84 / 145)$ \\
\hline Weight, kg & $n=121$ & $n=124$ & $n=121$ \\
\hline Mean \pm SD & $29.1 \pm 20.9$ & $30.6 \pm 24.7$ & $26.6 \pm 19.0$ \\
\hline Median (range) & 22.6 (3.0 to 83.7 ) & 21.3 (2.9 to 93.0$)$ & 21.0 (3.0 to 71.2$)$ \\
\hline BMI & $n=103$ & $n=109$ & $n=100$ \\
\hline Mean \pm SD & $17.7 \pm 4.1$ & $18.5 \pm 5.3$ & $18.2 \pm 4.4$ \\
\hline Median (range) & 17.1 (10.0 to 30.9 ) & 16.5 (11.6 to 33.6$)$ & 17.2 (10.0 to 31.9 ) \\
\hline CTAS score (out of 5) & $n=137$ & $n=135$ & $n=143$ \\
\hline Mean \pm SD & $2.9 \pm 0.6$ & $2.9 \pm 0.6$ & $2.8 \pm 0.7$ \\
\hline Median (range) & 3 (2 to 5$)$ & $3(2$ to 5$)$ & 3 (1 to 5$)$ \\
\hline Mode & 3 & 3 & 3 \\
\hline $\begin{array}{l}\text { Topical anesthetic cream } \\
\text { applied, \% }(n / N)\end{array}$ & $48(66 / 137)$ & $54(73 / 135)$ & $53(78 / 146)$ \\
\hline
\end{tabular}


comparisons were statistically significant in any of the outcomes (Table 2).

\section{Effect on secondary outcomes}

The mean number of attempts did not differ significantly between the study arms for the overall population or when stratified by age (Table 3 ). Successful placement was achieved in $70.6 \%$ of the population on the first attempt; $88.8 \%$ required up to 2 attempts, $97.1 \%$ needed up to 3 attempts, and $2.9 \%$ required 4 or more attempts for successful catheter placement.

Overall, the mean time from placement of tourniquet to success of catheterization did not differ significantly across the study arms (mean $8.3 \pm 15.9$ min for ultrasound imaging, $7.8 \pm 11.0$ min for near-infrared imaging and $6.5 \pm 10.3 \mathrm{~min}$ for standard approach; $p=0.5$ ). There were significantly fewer needle redirections with the standard approach $(1.6 \pm 3.2)$ than with ultrasound $(2.5 \pm 4.2)$ or near-infrared imaging $(2.8 \pm 5.0)$.

In an a priori logistic regression, the odds of first-attempt success was not associated with number of years as a nurse (odds ratio [OR] 0.98, $95 \%$ CI 0.94 to 1.01 , per additional year), number of years as an emergency nurse (OR 0.96, $95 \%$ CI 0.91 to 1.01 , per additional year), usual clinical emergency department time (OR 0.93,
95\% CI 0.78 to 1.12 , per additional hour) or patient's BMI (OR 0.99, $95 \%$ CI 0.98 to 1.00 , per additional $1 \mathrm{~kg} / \mathrm{m}^{2}$ ).

To explore whether results differed among nurses who participated in the trial procedures more frequently than others, we re-analyzed the primary outcome in the subset of nurses who performed the procedure more than 7 times $(n=$ 17). First-attempt success rates did not differ significantly and were similar to the primary analysis (ultrasound imaging v. standard approach: $4.4 \%, 95 \%$ CI $-8.3 \%$ to $17.1 \%$; near-infrared imaging v. standard approach: $-4.9 \%, 95 \% \mathrm{CI}$ $-18.3 \%$ to $8.5 \%$ ). In addition, in the analysis adjusted for clustering, we found no significant association between frequency of nurse participation and the primary outcome $(p=0.4)$.

\section{Interpretation}

We found that neither of the venous imaging devices offered an advantage over the standard approach to peripheral intravenous catheterization, even in the younger stratum. In fact, among children 3 years or younger, near-infrared imaging performed significantly worse than the standard approach based on differences in proportions. When we examined whether either intervention

Table 2: Proportion of patients with successful catheter placement on first attempt

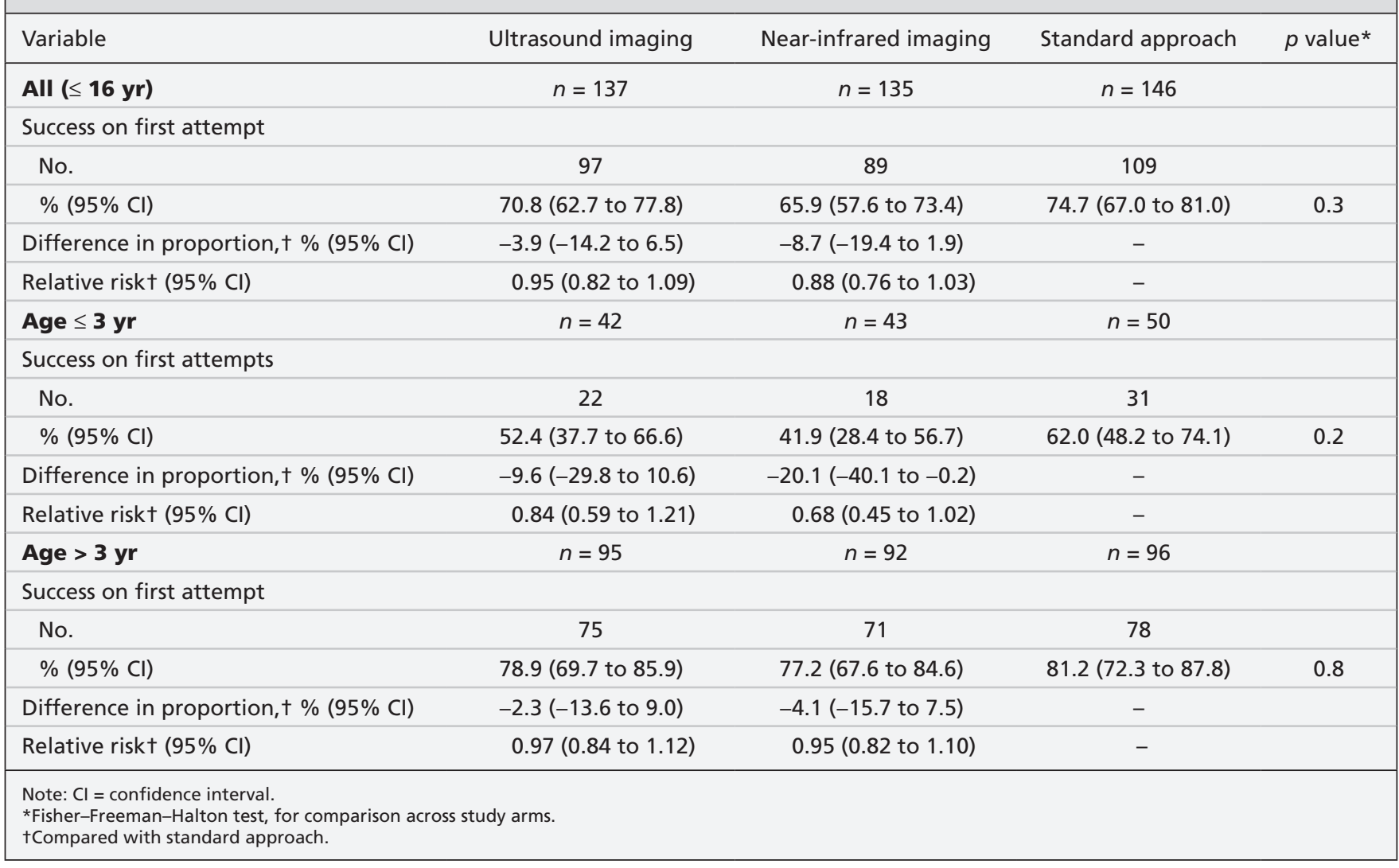


might be beneficial in children with increased adiposity, we found no association between BMI and first-attempt success rates in the logistic regression analysis. It was presupposed that nursing experience might positively influence rates of success; however, regression analysis revealed that success rates did not differ with increasing nursing experience in any group. Because randomization and performance of the procedure were in rapid succession, we did not have any crossover between study arms for the primary outcome; therefore, the intention-to-treat analysis is also per protocol.

Our randomized controlled trial was a direct comparison of near-infrared and ultrasound technologies because each had distinctive features that would plausibly facilitate improved intravenous catheterization over the standard technique. Direct comparison studies minimize bias and provide more conclusive results than between-study comparisons.

Reflecting standard staffing realities of a large urban emergency department, the trial had 83 nurses participating in it. Although it is conceivable that results could differ with a smaller number of nurses who are highly skilled in the use of adjunctive ultrasound for intravenous catheterization, expectations of the routine clinical availability of such professionals is not realistic in our busy acute care setting currently. As ultrasound devices become more user friendly and as nurses continue to train with new technologies, re-examination of ultrasound imaging by nurses for peripheral intravenous catheterization may be warranted in the future.

Most research on ultrasound imaging for peripheral intravenous catheterization has involved ultrasound-trained physicians and included adult

\begin{tabular}{|c|c|c|c|c|c|}
\hline \multirow[b]{2}{*}{ Variable } & \multicolumn{3}{|c|}{ Study arm; no. (\%) of patients* } & \multirow{2}{*}{$\begin{array}{c}\text { Mean difference } \\
(95 \% \mathrm{Cl}), \text { ultrasound } \\
\text { imaging - standard } \\
\text { approach }\end{array}$} & \multirow{2}{*}{$\begin{array}{c}\text { Mean difference } \\
(95 \% \mathrm{Cl}) \text {, near-infrarec } \\
\text { imaging - standard } \\
\text { approach }\end{array}$} \\
\hline & $\begin{array}{l}\text { Ultrasound } \\
\text { imaging }\end{array}$ & $\begin{array}{l}\text { Near-infrared } \\
\text { imaging }\end{array}$ & $\begin{array}{l}\text { Standard } \\
\text { approach }\end{array}$ & & \\
\hline All $(\leq 16$ yr) & $n=137$ & $n=135$ & $n=146$ & & \\
\hline Mean no. of attempts $\dagger$ & 1.40 & 1.58 & 1.43 & $-0.03(-0.23$ to 0.17$)$ & 0.14 (-0.10 to 0.38$)$ \\
\hline Median no. & 1 & 1 & 1 & & \\
\hline \multicolumn{6}{|l|}{ No. of attempts, no. (\%) } \\
\hline 1 & $96(70.1)$ & $90(66.7)$ & $109(74.7)$ & & \\
\hline 2 & $28(20.4)$ & $22(16.3)$ & $26(17.8)$ & & \\
\hline 3 & $11(8.0)$ & $19(14.1)$ & $5 \quad(3.4)$ & & \\
\hline$\geq 4$ & $2(1.5)$ & $4(3.0)$ & $6 \quad(4.1)$ & & \\
\hline Age $\leq \mathbf{3}$ yr & $n=42$ & $n=43$ & $n=50$ & & \\
\hline Mean no. of attempts & 1.69 & 2.10 & 1.76 & $-0.05(-0.55$ to 0.44$)$ & $0.33(-0.25$ to 0.92$)$ \\
\hline Median no. & 1.5 & 2 & 1 & & \\
\hline \multicolumn{6}{|l|}{ No. of attempts, no. (\%) } \\
\hline 1 & $21(50.0)$ & $19(44.2)$ & $31(62.0)$ & & \\
\hline 2 & $14(33.3)$ & $11(25.6)$ & $12(24.0)$ & & \\
\hline 3 & $6(14.3)$ & $10(23.3)$ & $3(6.0)$ & & \\
\hline$\geq 4$ & $1(2.4)$ & $3(7.0)$ & $4(8.0)$ & & \\
\hline Age $>\mathbf{3} \mathbf{y r}$ & $n=95$ & $n=92$ & $n=96$ & & \\
\hline Mean no. of attempts§ & 1.28 & 1.36 & 1.26 & $-0.02(-0.20$ to 0.15$)$ & $0.08(-0.12$ to 0.28$)$ \\
\hline Median no. & 1 & 1 & 1 & & \\
\hline \multicolumn{6}{|l|}{ No. of attempts, no. (\%) } \\
\hline 1 & $75(78.9)$ & $71(77.2)$ & $78(81.2)$ & & \\
\hline 2 & $14(14.4)$ & $11(12.0)$ & $14(14.6)$ & & \\
\hline 3 & $5 \quad(5.3)$ & $9 \quad(9.8)$ & $2(2.1)$ & & \\
\hline$\geq 4$ & $1 \quad(1.1)$ & $1 \quad(1.1)$ & $2(2.1)$ & & \\
\hline $\begin{array}{l}\text { *Unless stated otherwise. } \\
\text { †No significant difference acr } \\
\text { ‡No significant difference acr } \\
\text { §No significant difference acr }\end{array}$ & $\begin{array}{l}\mathrm{ms}(p=0.2 ; \mathrm{Kr} \\
\mathrm{ms}(p=0.1 ; \mathrm{Kr} \\
\mathrm{ms}(p=0.7 ; \mathrm{Kr}\end{array}$ & $\begin{array}{l}\text { al-Wallis test). } \\
\text { a-Wallis test). } \\
\text { a-Wallis test). }\end{array}$ & & & \\
\hline
\end{tabular}


patients and more controlled settings. The extrapolation of these results to pediatrics and to the acute care setting (where most pediatric intravenous catheters are placed, by nurses) is questionable.

Heinrichs and colleagues ${ }^{14}$ described 2 small pediatric emergency department trials of ultrasound-guided peripheral intravenous catheterization, with physician as ultrasonographer and nurse as cannulator. One trial $(n=50)$ showed that dynamic or real-time use of the technology (locate vessel with ultrasound probe and simultaneously insert cannula) decreased the mean number of attempts by 2 and the mean procedure time by 8 minutes. ${ }^{12}$ The other trial $(n=44)$ did not show a clinically important benefit to static use of the technology (locate and mark vessel, discard ultrasound probe and insert cannula). ${ }^{13}$ We took a pragmatic approach in our large trial: nurses, who both applied the technology and performed the catheterization, were trained in both dynamic and static techniques and were asked to choose whichever technique was judged at the bedside to provide the best likelihood of success for each attempt. Despite this rational approach to use of the technology, our results did not support use of either technology as an adjunctive tool for the procedure.

Assessment of our results in light of other randomized trials of near-infrared technologies similar to the VeinViewer device revealed a similar direction of effect. Overall, trials of nearinfrared technologies in emergency department ${ }^{7,8}$ and operating room settings ${ }^{15-17}$ have not shown a convincing clinical benefit of near-infrared imaging for peripheral intravenous catheterization relative to the standard approach. ${ }^{18}$

\section{Limitations}

Given the size and obvious presence of the devices studied, complete concealment of group allocation was not possible. Thus, performance bias against the technologies may have influenced the results. However, nurses who were not enthusiastic about the new technologies could decline to participate in the trial. Therefore, participating nurses were open to the idea that use of the technologies could improve the success rate of intravenous catheter placement on first attempt and were unlikely to be negatively biased against the interventions. In addition, lack of blinding is more problematic when outcome measures are subjective or surrogate. Our outcome measures were objective and clinically relevant. Blinding occurred at the level of the research team and the statistician.

The nurses may not have been trained well enough in the use of the devices. The training was comprehensive and comparable to that in other similar trials, and we required that participating nurses declare confidence in using both technologies for the procedure and show proficiency on gel models for the ultrasound imaging. However, we did not require a demonstration of proficiency through a preset formal assessment on patients. Ultrasound imaging may not be the type of technology that can be adapted easily without focused training and perhaps a natural proclivity. Much is unknown about characteristics of proficient learners of this technology, and training needs likely differ across learners.

The study population was recruited based on the availability of research staff and thus was a convenience sample. However, staffing was tailored to cover the busiest emergency department times, and study logs with details about missed patients described typical pediatric emergency patients consistent with those recruited.

\section{Conclusion}

We found no evidence that ultrasound or nearinfrared imaging improved the success rates of peripheral intravenous catheterization on first attempt in a pediatric emergency department. In addition, neither technology reduced the number of catheterization attempts or increased the speed of the procedure. These 2 widely available technologies do not appear to help usual-skilled clinicians complete this stressful procedure better than the standard approach. Thus, current evidence does not support investment in these technologies for the purpose of routine peripheral intravenous catheterization in children.

The results of our trial underscore the importance of rigorously examining new technologies in children before adopting them into clinical care. Not doing so may lead to a false understanding of their benefits and limitations, which may mislead clinicians, administrators and patients alike.

\section{References}

1. Welch SJ. Twenty years of patient satisfaction research applied to the emergency department: a qualitative review. Am J Med Qual 2010;25:64-72.

2. Lininger RA. Pediatric peripheral i.v. insertion success rates. Pediatr Nurs 2003;29:351-4.

3. Reigart JR, Chamberlain KH, Eldridge D, et al. Peripheral intravenous access in pediatric inpatients. Clin Pediatr (Phila) 2012; 51:468-72.

4. Peterson KA, Phillips AL, Truemper E, et al. Does the use of an assistive device by nurses impact peripheral intravenous catheter insertion success in children? J Pediatr Nurs 2012;27:134-43.

5. Frey AM. Success rates for peripheral IV insertion in a children's hospital. J Intraven Nurs 1998;21:160-5.

6. Black KJ, Pusic M, Harmindy D, et al. Pediatric intravenous insertion in the emergency department. Pediatr Emerg Care 2005; 21:707-11.

7. Chapman LL, Sullivan B, Pacheco AL, et al. VeinViewerassisted intravenous catheter placement in a pediatric emergency department. Acad Emerg Med 2011;18:966-71.

8. Perry AM,Caviness AC, Hsu DC. Efficacy of a near-infrared light device in pediatric intravenous cannulation: a randomized controlled trial. Pediatr Emerg Care 2011;27:5-10.

9. Katsogridakis YL, Seshadri R, Sullivan C, et al. Veinlite transillumination in the pediatric emergency department: a therapeutic interventional trial. Pediatr Emerg Care 2008;24:83-8.

10. Goff DA, Larsen P, Brinkley J, et al. Resource utilization and 
cost of inserting peripheral intravenous catheters in hospitalized children. Hospital Pediatrics. 2013;3:185-91.

11. Rinke ML. Not just a little pinch: first do no harm with pediatric peripheral IV catheters. Hosp Pediatr 2013;3:192-3.

12. Doniger SJ, Ishimine P, Fox JC, et al. Randomized controlled trial of ultrasound-guided peripheral intravenous catheter placement versus traditional techniques in difficult-access pediatric patients. Pediatr Emerg Care 2009;25:154-9.

13. Bair AE, Rose JS, Vance CW, et al. Ultrasound-assisted peripheral venous access in young children: a randomized controlled trial and pilot feasibility study. West J Emerg Med 2008;9:219-24.

14. Heinrichs J, Fritze Z, Vandermeer B, et al. Ultrasonographically guided peripheral intravenous cannulation of children and adults: a systematic review and meta-analysis. Ann Emerg Med 2013;61:444-454.e1.

15. Cuper NJ, De Graaff JC, Verdaasdonk RM, et al. Near-infrared imaging in intravenous cannulation in children: a cluster randomized clinical trial. Pediatrics 2013;131:e191-7.

16. Szmuk P, Steiner J, Pop RB, et al. The VeinViewer vascular imaging system worsens first-attempt cannulation rate for experienced nurses in infants and children with anticipated difficult intravenous access. Anesth Analg 2013;116:1087-92.

17. de Graaff JC, Cuper NJ, Mungra RAA, et al. Near-infrared light to aid peripheral intravenous cannulation in children: a cluster randomised clinical trial of three devices. Anaesthesia 2013;68:835-45

18. Heinrichs J, Fritze Z, Klassen T, et al. A systematic review and meta-analysis of new interventions for peripheral intravenous cannulation of children. Pediatr Emerg Care 2013;29:858-66.

Affiliations: Department of Pediatrics (Curtis, Craig, Logue), University of Alberta; Alberta Research Centre for Health Evidence (Vandermeer); Department of Emergency Medicine (Hanson), University of Alberta; Women and Children's Health Research Institute (Curtis, Craig), Edmonton, Alta; Manitoba Institute of Child Health and College of Medicine (Klassen), Faculty of Health Sciences, University of Manitoba, Winnipeg, Man.
Contributors: Sarah Curtis conceived the study. She designed the trial with Terry Klassen and William Craig, with contributions from Erin Logue and Amanda Hanson. Sarah Curtis supervised the conduct of the trial and data collection, and she coordinated the trial and managed the data with William Craig. Ben Vandermeer provided statistical advice on the study design and analyzed the data. Sarah Curtis drafted the manuscript, and all of the authors contributed substantially to its revision. All of the authors approved the final version to be published and agreed to act as guarantors of the work.

Funding: This research was funded by the Women and Children's Health Research Institute through the generous support of the Stollery Children's Hospital Foundation. The funding agency had no role in the study design, the interpretation of data, the manuscript preparation or the decision to publish.

Data sharing: Data are available upon request to the corresponding author. Data can be accessed after a signed data transfer agreement is in place. Data will be transferred electronically through a password-protected file.

Acknowledgements: The authors thank the participating families and the clinical and research staff at the Stollery Children's Hospital for their support of and participation in this study. They also acknowledge the research coordination provided by Joyce Chickuma and thank Dr. Robert Evans for his critical review of the manuscript and technical support. The Clinical Research Informatics Core at the Women and Children's Research Institute provided support for data management. Statistical support was provided by the Evidence-based Practice Center, University of Alberta, Edmonton, Alta. 